\title{
In Vivo Characterization of Ultrasonic Transducers for the Detection of Airway Occlusion in Sleep Disordered Breathing
}

\author{
Mohammad A. Al-Abed, Student Member, IEEE, Peter Antich, Senior Member, IEEE, \\ Donald E. Watenpaugh, and Khosrow Behbehani, Senior Member, IEEE
}

\begin{abstract}
Obstructive sleep apnea/hypopnea Syndrome (OSAHS) is the most common form of Sleep Disordered Breathing (SDB) and it is estimated to affect approximately $15 \%$ of US adult population. Various methods have been proposed for the development of inexpensive screening methods to detect SDB to reduce the need for costly nocturnal polysomnography (NPSG). In this paper, a description of the ultrasonic transducer design and characterization is presented, followed by the results of a full night sleep study. The findings show a significant difference in the temporal features extracted from the received ultrasonic waveform during apneic breathing, compared to the hyperventilation that follows. Therefore, the findings indicate the feasibility of developing an ultrasonic detection device for low cost diagnosis of SDB.
\end{abstract}

\section{INTRODUCTION}

$\mathrm{O}$ BSTRUCTIVE Sleep Apnea/Hypopnea Syndrome (OSAHS) is a sleep disorder, characterized by repetitive pharyngeal collapse. It has been recently reported that prevalence of untreated sleep apnea is approximately $15 \%$ of the United States adult population [1], heightening the necessity for more wide spread screening. Patients diagnosed with OSAHS have high incidence of obesity, and exhibit an increased risk of hypertension, stroke, and depression. An association between OSAHS and high morbidity and mortality due to cardiovascular and cerebrovascular causes has been established [1-4].

Obstructive apneas consist of complete occlusion of the airway at the pharynx, causing a cessation of airflow for ten seconds or more with continuing respiratory effort despite the occlusion. Hypopnea is manifested by a decrease of $50 \%$ or more in the airflow for ten seconds or more, due to the narrowing of the airway at the pharynx [5]. An average of five events or more in an hour of sleep is considered clinically significant, and therefore worthy of medical intervention. This average measurement is called the

Manuscript received April 15, 2011. This work was supported in part by a grant from the U.S. Department of Energy.

M. A. Al-Abed is a Ph.D. student with the Department of Bioengineering, the University of Texas at Arlington, Arlington, TX 76010 USA (phone: 817-272-2249; fax: 817-272-2251; e-mail: mohammad@uta.edu).

P. Antich is professor and chair of the Radiology Department at the University of Texas Southwestern Medical Center in Dallas, Dallas TX 75390 USA (e-mail: Peter.Antich@UTSouthwestern.edu).

D. E. Watenpaugh is a physiologist and sleep medicine specialist with Sleep Consultants, Inc., Fort Worth, TX, 76104 USA.

K. Behbehani is a professor and chair of the Department of Bioengineering Department, the University of Texas at Arlington, Arlington, TX 76010 USA (e-mail:kb@uta.edu).
Apnea/Hypopnea Index (AHI) [4]

Nocturnal polysomnography (NPSG) performed in accredited sleep laboratories is the gold standard for diagnosis of OSAHS. However, due to the cost of such test and lack of sleep laboratories in all areas, low cost, yet reliable methods for diagnosis of SDB are highly desired. The current methods that have been developed for diagnosis and large-scale screening for SDB have focused on markers of physiological responses associated with events of airway occlusion, such as sympathoexcitatory or cardiovascular reactions.

We have reported in [6] an in vitro study of ultrasonic sensors for the detection of occlusion in an upper airway model. There are several advantages in using ultrasonic in detecting OSAHS: it is safe (non-ionizing radiation, no contrast agent), non-invasive, low cost, and portable [7]. In this paper, we report preliminary results of an in vivo study for the detection of airway occlusion during apnea/hypopnea events using ultrasonic transducers. The ultrasonic transducer design parameters, anatomical considerations, and beam profile characterization is evaluated. In vivo experiment protocol and preliminary results are presented.

\section{II.PRELIMINARY DESIGN CONSIDERATIONS}

\section{A. Site of Airway Obstruction}

The site of occlusion of the airway during obstructive sleep apnea events differs slightly, with intra- and inter-subject variations. However, most studies, irrespective of the technique used to localize the occlusion, indicate that the main site of occlusion is the oropharynx, sometimes with extension to the laryngopharynx [8]. Figure 1 shows an illustration of the sagittal view of the head and neck, showing the upper airway and its segments. The oropharynx is the segment of the airway that extends from the end of hard palate to the epiglottis, and includes the soft palate and posterior tongue [8].

\section{B. Anatomical and Practical Considerations for the Ultrasonic Transducer Design}

In order to design skin-surface, non-invasive ultrasonic transducers for the purposes of detecting the presence of occlusion in the upper airway during apneic breathing, the following design consideration must be addressed:

1. The heterogeneity of the media (i.e. skin, fat, muscle, bone, cartilage, air, etc.) must be considered. 


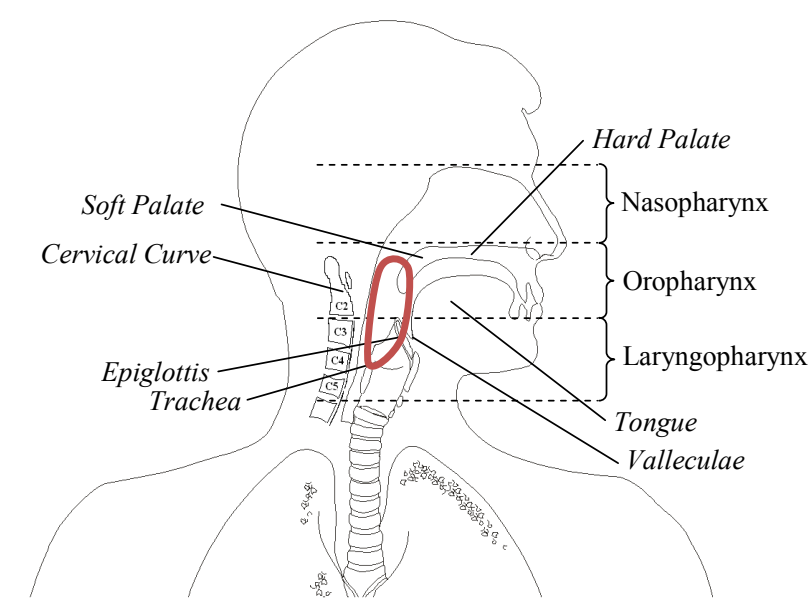

Fig. 1. Sagittal illustration of the head and neck showing the anatomy of the airway. The marked area represents the portion of upper airway (oropharynx, with extension to the laryngopharynx) where the occlusion occurs during obstructive sleep apnea events.

2. Considering the neck curvature, the transducers must have a small surface (i.e. small footprint), while being large enough for handling and assembly.

3. The curvature of the neck also requires the receivers' ability to detect the ultrasonic pulses at either normal or oblique incidence.

4. The main site of occlusion differs between patients, which requires a longitudinal array of transducers, pulsed simultaneously, to capture the occlusion when it occurs, and to allow for reception spatial resolution to estimate the location of occlusion.

\section{MethodOLOGY}

\section{A. Transmitter Transducer Design}

In order to achieve maximum acoustic energy penetration through the neck, a low frequency and large surface area transducer is ideal [9]. However, since the thickness of a piezoelectric transducer, such as those made of leadzirconate-titanate (PZT) crystals, is equal to half the wavelength [10], the crystal thickness doubles as the frequency is halved as seen from (1):

$$
\lambda \cdot f=c
$$

where $\lambda$ is the wavelength [m], $f$ is the sensor frequency $[\mathrm{Hz}]$, and $c$ is the speed of the sound in the medium $[\mathrm{m} / \mathrm{s}]$. Also, a transducer with large surface area would be difficult to have full skin contact during the night due to the curvature of the neck.

To address these limitations, a low-Q disk piezoelectric, with a central frequency of $3 \mathrm{MHz}$ was chosen for this design, and a radius of $a=5 \mathrm{~mm}$. The thickness of the PZT crystal is $0.58 \mathrm{~mm}$, for $f=3 \mathrm{MHz}$ and $c=4350 \mathrm{~m} / \mathrm{s}[9,10]$.

The PZT has an acoustic impedance of $Z_{o}=33.7$ MRayl. Brass (acoustic impedance of $Z=29.5 \mathrm{MRayl}$ ) was used as a backing layer.

The characterization of the beam profile for the transmitter can be found by calculating and plotting the far- field pressure directivity function. The Fraunhofer approximation of the directivity function of a disk transducer, using continuous wave $(\mathrm{CW})$ excitation, $D_{\text {circ }}(\theta)$, can be calculated as $[10]$ :

$$
D_{\text {circ }}(\theta)=2 \cdot J_{l}(k \cdot a \cdot \sin \theta) /(k \cdot a \cdot \sin \theta)
$$

where $J_{l}$ (.) is the Bessel function of the $1^{\text {st }}$ kind, $k=2 \pi / \lambda$ is the wave number in the medium, $a$ is the sensor radius, and $\theta$ is in radians, and measures of the angular displacement normal to the transducer surface.

Using (2), the directivity function for the disk transducer is found and shown in Figure 2. The divergence half angle, $\theta_{R}$, is found to be $3.5^{\circ}$. This is the angle that the main lobe is enclosed in. The smaller this angle is, the higher the transducer's directivity.

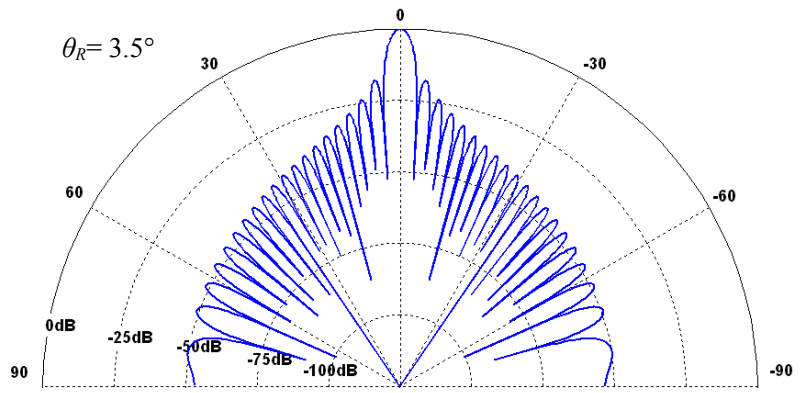

Fig. 2. This figure the directivity function, $D(\theta)$, for the disk transducer, in $\mathrm{dB}$, for $-\pi / 2<\theta<\pi / 2$. Notice that the directivity function is not dependent on the distance between the observation point and the transducer; it is a function of the angle of the observation point in reference to the axis normal to the surface of the transducer.

\section{B. Receiver Transducer Design}

The design considerations for the receiver transducers are similar to those for the transmitter. However, in order to achieve maximum reception, these transducers need to be designed with a wider beam width to allow for normal and oblique incidence of the received ultrasonic waveform, to account for the curvature of the neck. Small size elements are desirable for this purpose; however, assembly and handling becomes an issue for very small transducers.

In order to meet these design requirements, rectangular transducers were designed with width $W=4 \mathrm{~mm}$, and height $H=10 \mathrm{~mm}$. Following the reciprocity concept, the characterization of the reception field of these transducers can be calculated using the beam profile for the element. The far-field Fraunhofer approximation of the directivity function of a rectangular transducer, using continuous wave $(\mathrm{CW})$ excitation, $D_{\text {rect }}(\theta, \varphi)$, can be calculated as [10]:

$$
D_{\text {rect }}(\theta, \varphi)=\operatorname{sinc}((W / \lambda) \cdot \sin \theta) \times \operatorname{sinc}((H / \lambda) \cdot \sin \varphi)
$$

where; $\lambda$ is the wave length, $W$ is the element width, $H$ is the element height, $\theta$ is in radians, and measures of the angular displacement along the width axis normal to the transducer surface, and $\varphi$ is in radians, and measures of the angular displacement along the height axis normal to the transducer surface.

The receiver transducers match the transmitter frequency at $3 \mathrm{MHz}$. The thickness of the transducer is $0.58 \mathrm{~mm}$ and a brass layer was used as a backing layer. 
Using (3), the directivity function for the rectangular element along the $\theta=0^{\circ}$ axis (the $H$-axis) is found and shown in Figure 3 (A). The divergence half angle, $\varphi_{R}$, is found to be $2.7^{\circ}$. The directivity function along $\varphi=0^{\circ}$ axis (the $W$-axis) is also found and shown in Figure 3 (B). The divergence half angle, $\theta_{R}$, is found to be $6.8^{\circ}$.
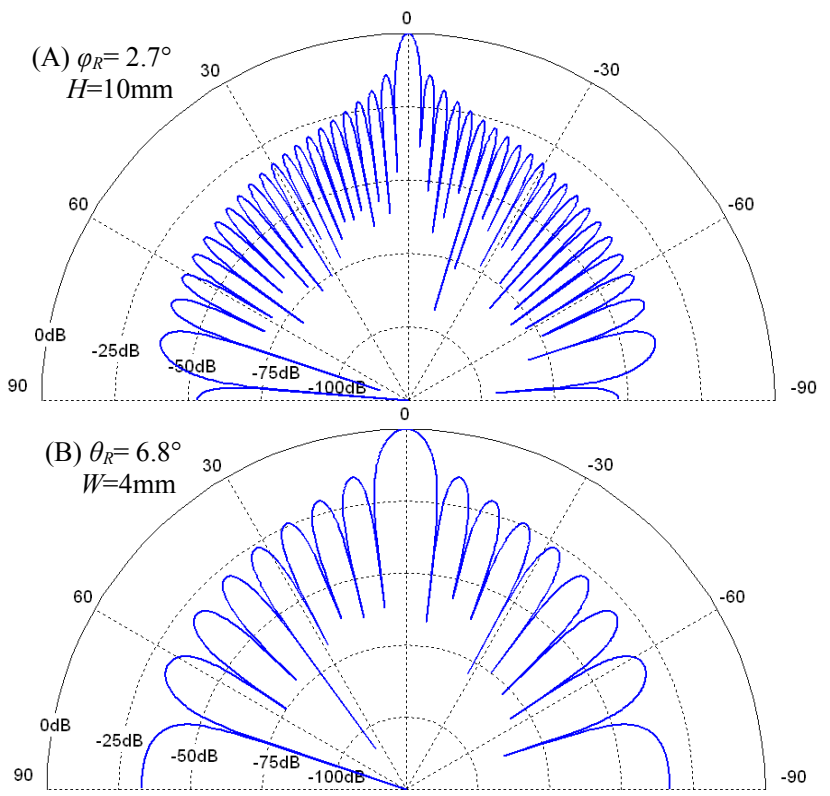

Fig. 3. Shows the directivity function, $D(\theta, \varphi)$, for the rectangular element along (A) the height (for $\theta=0^{\circ}$ ) and (B) the width (for $\varphi=0^{\circ}$ ). Notice that the directivity function is not dependent on the distance between the observation point and the transducer; it is a function of the angle of the observation point in reference to the axis normal to the surface of the transducer.

\section{In Vivo Experiment Design}

To test the ultrasonic transducer assembly for in vivo detection of airway occlusion during apnea, a protocol for full night sleep study at an accredit sleep lab was submitted to and approved by the local IRB committee. The protocol calls for recruitment of patients previously diagnosed with SDB, but do not have other underlying neurological or physiological abnormalities, for a full night sleep (6-8 hours) at an accredited sleep lab (Sleep Consultants, Inc., Fort Worth, TX). A consent form was provided and signed by the volunteers. The patients underwent full NSPG, in simultaneously with the ultrasonic transducers placed on the patient's neck. The transducers are placed along the length of the neck below the earlobe and just behind the mandibular joint. The area covered by the transducers on either side of the neck is about $2.5 \mathrm{~cm} \times 8.0 \mathrm{~cm}$.

The data acquisition system used for the ultrasound experimental setup is described in [6]. The transmitters were simultaneously pulsed at 10 Pulse Repetition Frequency $(\mathrm{PRF}=10)$ using OmniScan ${ }^{\circledR} i X$ UT (Olympus NDT, Quebec, Canada). The transmitted and received signals from both transducer assemblies are acquired using Data Acquisition (DAQ) system (National Instruments NI PXI-5105, Austin, TX, USA) running at $30 \times 10^{6}$ Samples/s, 12-bits/Sample.
At the end of the study, a certified sleep expert, blind to the aims of this study, scored the NPSG data according to Rechtschaffen \& Kales standard. The severity of SDB was measured using the apnea-hypopnea index (AHI).

\section{D.Post Processing}

Each second, there are 10 received pulses. The received signal is summed for each second into one waveform. This waveform is then filtered using a Kaiser Window band-pass FIR filter $\left(f_{L}=0.15 \mathrm{MHz}, f_{H}=3.5 \mathrm{MHz}, N=100, \beta=0.05\right)$ and rectified. The peaks of the resultant signal are detected, and an envelope of the peaks is estimated using a cubic spline.

\section{E. Waveform Envelope Feature Extraction}

For each envelope, three temporal features were extracted:

1. Peak: The maximum value of the envelope is detected and recorded.

2. Area: The area under the curve is calculated and recorded.

3. Location: The temporal location, at which the maximum value of the envelope occurs, with reference to the time that a pulse is applied, is detected and recorded.

\section{F. Respiratory Event Epoch Clipping}

Each respiratory event (RE), whether it is apnea or hypopnea, is followed by a period of hyperventilation (HV). The aim is to quantify the changes in the received signal between RE and HV, using the temporal features extracted above. Each RE that is followed by 10 seconds of $\mathrm{HV}$ is clipped and considered for analysis.

The average peak, area, and location for each RE can be calculated, and are denoted $\mu_{P E A K, n}^{R E}, \mu_{A R E A, n}^{R E}$ and $\mu_{L O C, n}^{R E}$, respectively. The average peak, area, and location for each $\mathrm{HV}$ that follows each RE can be calculated, and are denoted $\mu_{P E A K, n}^{H V}, \quad \mu_{A R E A, n}^{H V}$ and $\mu_{L O C, n}^{H V}$ respectively. Notice that $n$ denotes the index of the clipped epoch $1 \leq \mathrm{n} \leq q$ where $q$ is the total number of epochs used in the analysis.

\section{G.Recruited Patient Demographics}

One male volunteer was recruited (49 years old, BMI = $34.9 \mathrm{~kg} / \mathrm{m}^{2}, \mathrm{AHI}=86.3$ ). Duration of sleep study was 7 hours. There were $q=369$ epochs that met the criteria described above. From those, 197 were apnea events, and 172 hypopnea events. AHI).

\section{H.Statistical Analysis}

The ratio of the average of peaks in the HV period to the average of peaks in the RE for each epoch was calculated; that is, the ratio $R P_{n}=\mu_{P E A K, n}^{H V} / \mu_{P E A K, n}^{R E}$ is found, where $R P$ denotes Ratio of Peaks. Similarly, Ratio of Areas and Ratio of Location were calculated $R A_{n}=\mu_{A R E A, n}^{H V} / \mu_{A R E A, n}^{R E}$ and $R A_{n}=\mu_{L O C, n}^{H V} / \mu_{L O C, n}^{R E}$.

The $95 \%$ confidence interval (CI) is calculated using the $t$ distribution as follows [11]: 


$$
R_{\text {feature }}^{C I}=\overline{R_{\text {feature }}} \pm\left(t_{0.05, q} \times \sigma_{\text {feature }} / \sqrt{ } \mathrm{q}\right)
$$

where $\overline{R_{\text {feature }}}$ is the mean of $R P, R A$, or $R L ; \sigma_{\text {feature }}$ is the standard deviation of $R P, R A$, or $R L ; q$ is the number of epochs used ( $q=197$ for apnea, $q=172$ for hypopnea, and $q=369$ for both combined); and $t_{0.05, q}$ is the two-tail critical t-value for a $95 \%$ confidence interval and $q$ degrees of freedom (df). It is about 1.97 for the $q$ values listed above.

\section{RESULTS}

The confidence interval for the epochs containing apnea, hypopnea, and the combination of both are plotted in Figure 4. Notice that if the confidence interval does not include 1, it indicates that there is a significant difference between the average feature values between $\mathrm{RE}$ compared to the subsequent $\mathrm{HV}$.

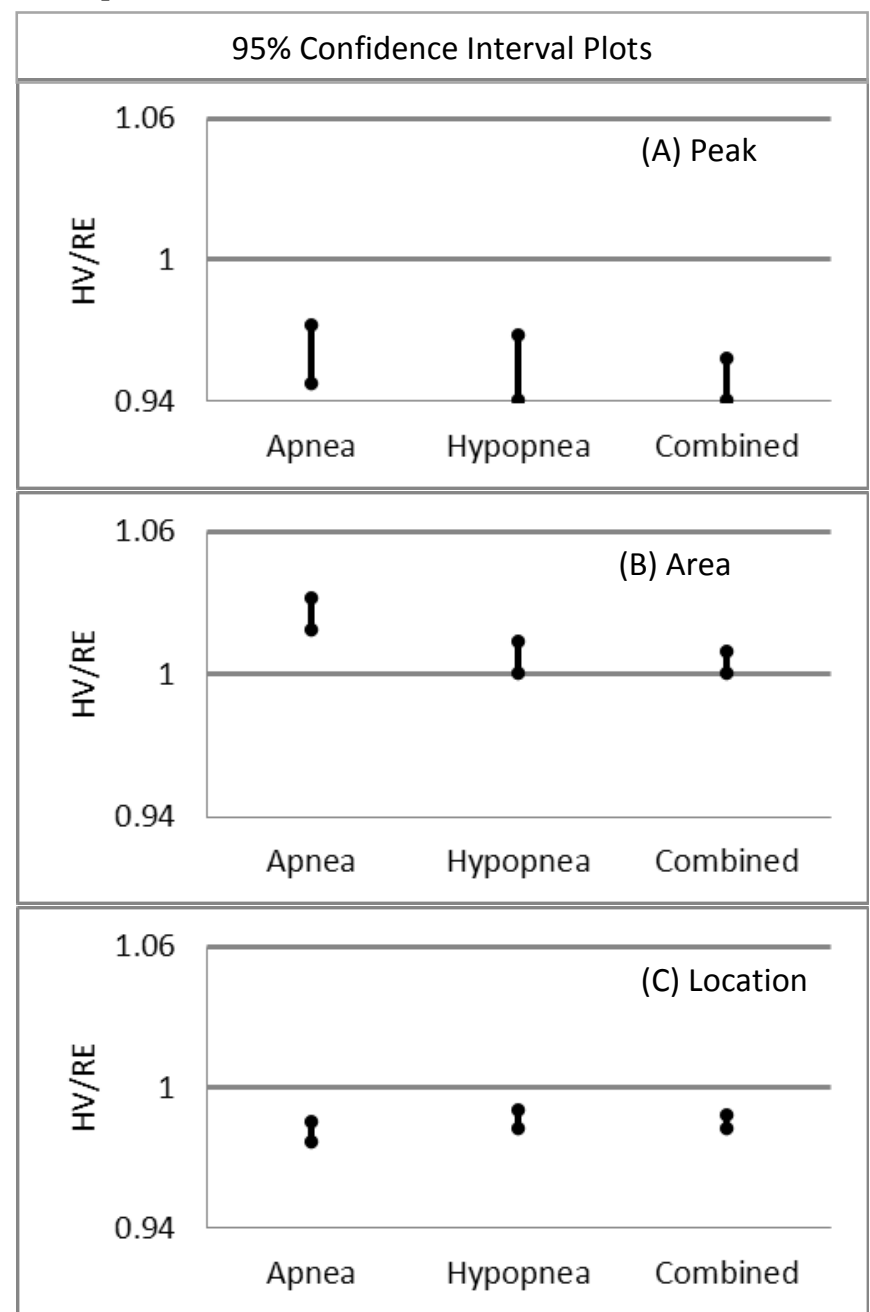

Fig. 4. The $t$-distribution 95\%-Confidence Interval plots of the (A) Peak, (B) Area, and (C) Location for apnea and hypopnea epochs, and the combination of both epoch groups. HV/RE denotes the mean ratio of the average said feature between hyperventilation (HV) and the superseding respiratory event $(\mathrm{RE})$.

\section{V.DISCUSSION}

In this in vivo feasibility study for the use of ultrasonic pulses to detect the closure of the upper airway during apneic breathing, the ultrasonic transducer design followed a set of anatomical and practical considerations for overnight ultrasonic data acquisition. From studying Figure 2, the transmitter disk element shows high directivity and concentrated energy to penetrate deep through the neck. By contrast, the rectangular elements have a wider beam profile, as shown in Figure 3, allowing reception at a wide range of incidence beam angles.

Studying the epochs recorded using the ultrasonic transducers during the full night sleep study; we notice that temporal features extracted from these records differ between apneic breathing and the hyperventilation period that follows. In particular, we notice a significant difference between the average peaks, areas, and locations between apneic breathing and the succeeding period of hyperventilation.

\section{CONCLUSION}

This paper presented a novel in vivo study for the utilization of ultrasonic sensing for the non-invasive detection of airway narrowing and occlusion. Using skinmounted ultrasound transducer elements, an examination of the possibility of distinguishing apneic breathing from normal breathing was performed using epochs collected from one SDB volunteer. While the results presented here are preliminary, the use of ultrasound detection of airway occlusion events may offer a simple, cost effective, and specific screening tool for OSAHS breathing pattern.

\section{REFERENCES}

[1] T. Young, M. Palta, J. Dempsey, P. E. Peppard, F. J. Nieto, and K. M Hla, "Burden of sleep apnea: rationale, design, and major findings of the Wisconsin Sleep Cohort study," Wisconsin Medical Journal, vol. 108, pp. 246-249, 2009.

[2] P. J. Strollo and R. M. Rogers, "Obstructive Sleep Apnea," New England Journal of Medicine, vol. 334, pp. 99-104, 1996.

[3] T. Young, L. Finn, P. E. Peppard, M. Szklo-Coxe, D. Austin, F. J Nieto, R. Stubbs, and K. M. Hla, "Sleep Disordered Breathing and Mortality: Eighteen-Year Follow-up of the Wisconsin Sleep Cohort," Sleep, vol. 31, pp. 1071-1078, 2008.

[4] T. Young, P. E. Peppard, and D. J. Gottlieb, "Epidemiology of Obstructive Sleep Apnea: A Population Health Perspective," American Journal of Respiratory and Critical Care Medicine, vol. 165, pp. 1217-1239, 2002.

[5] F. Roux, C. D’Ambrosio, and V. Mohsenin, "Sleep-related breathing disorders and cardiovascular disease," The American Journal of Medicine, vol. 108, pp. 396-402, 2000.

[6] M. Al-Abed, P. Antich, D. E. Watenpaugh, and K. Behbehani, "Detection of airway occlusion in simulated obstructive sleep apnea/hypopnea using ultrasound: An in vitro study," presented at the 32nd Annual International Conference of the IEEE Engineering in Medicine and Biology Society, Buenos Aires, Argentina, 2010.

[7] J. Quistgaard, "Signal acquisition and processing in medical diagnostic ultrasound," IEEE Signal Processing Magazine, vol. 14, pp. 67-74, 1997.

[8] A. N. Rama, S. H. Tekwani, and C. A. Kushida, "Sites of Obstruction in Obstructive Sleep Apnea," Chest, vol. 122, pp. 1139-1147, 2002.

[9] W. R. Hedrick, D. L. Hykes, and D. E. Starchman, Ultrasound Physics and Instrumentation, Fourth ed. St. Louis, MO: Elsevier Mosby, 2005.

[10] R. S. Cobbold, Foundations of Biomedical Ultrasound, 1st ed. New York City, New York: Oxford University Press, 2007.

[11] J. H. Zar, Biostatistical Analysis, 4th Edition ed. Upper Saddle River, New Jersey: Prentic hall, 1999. 